RECORDS OF PHARMACEUTICAL
AND BIOMEDICAL SCIENCES

\title{
Colloidal delivery of drugs: present strategies and conditions
}

\author{
Nahed Sallam $^{a}$, Rania Sanad ${ }^{a}$, El-Sayed Khafagy $^{b}$, Ahmed Mahgoub $^{c}$, Mamdouh Ghourab $^{b}$, Shadeed Gad $^{b}$ \\ ${ }^{a}$ Department of Pharmaceutics, National Organization for Drug Control and Research, Egypt; ${ }^{b}$ Department \\ of Pharmaceutics, Faculty of Pharmacy, Suez Canal University, Egypt; ${ }^{c}$ Department of Molecular Drug \\ Evaluation, National Organization for Drug Control and Research, Egypt.
}

Received on: 25. 06. 2020

Revised on: 28. 07. 2020

Accepted on: 09. 08. 2020

*Correspondence Author:

Tel. : 201003934422

E-mail address:

shaded_abdelrahman@pharm.suez.edu.eg

\begin{abstract}
In this report, we give a description of a broad range of colloidal drug delivery systems with special focus on vesicles used in research or potentially useful as carrier systems for pharmaceutical drugs or active biomolecules or as therapeutic cell carriers. Colloidal drug delivery systems provide some essential descriptions of the drug delivery systems currently being built for academic or clinical applications. This series of systems are commonly used due to outstanding drug tracking, continuous and regulated release behavior, improved drug molecules clogging performance, avoidance of product hydrolysis or enzymatic degradation, and therapeutic efficacy improvements. Such characteristics aid in the discovery of suitable carrier structures for the transmission of medicines, cells, and genes in various fields.
\end{abstract}

Keywords: Vesicular drug delivery, noisome, bilosome, spanlastics.

\section{Introduction}

Starch Drug delivery challenges include poor drug solubility, poor bioavailability, peptide and protein delivery, large particle size, in vivo stability, intestinal absorption, sustained and targeted delivery to the site of action, therapeutic efficacy, generalized side effects, and drug plasma fluctuation (Kayser et al., 2005).
Such problems are solved by many modern drug delivery systems, using bioadhesive systems, transdermal patches, nanoscale devices, implants, microparticulate systems and even the path to delivery as nasal or buccal DDSs (de la Fuente et al., 2010). 
Nanotechnology offers advantages that allow drug delivery to be more

targeted and therapeutic compound release to be managed.

The key objective of nanotechnology is controlled drug delivery, i.e. regulation of drug release at the target site; pharmacokinetic, pharmacodynamic modulation, reduction of dosing frequency; reduction of toxicity, and improvement of efficacy

(Gupta et al., 2013; Singh et al., 2014).

Nanostructures have many advantages as the ability to shield drugs from gastrointestinal tract degradation; the technology enables direct delivery of drugs to specific areas of the body. They allow poorly water-soluble medicines to be administered by providing ways to sidestep the liver, thus avoiding metabolism in the first step.
They allow poorly water-soluble drugs which undergo first pass metabolism in the liver to sidestep this metabolism when administered orally (Kayser et al., 2005). Nanotechnology improves the oral bioavailability of drugs due to their advanced takeup mechanisms such as absorptive endocytosis and can remain in the blood for a long time, releasing the inserted drug in a regulated manner, resulting in fewer plasma variability and minimizing side effects (Gupta et al., 2013; Ameeduzzafar et al., 2016).

Products of nanoscale size can penetrate tissues and are quickly absorbed by cells, allowing for the efficient delivery of drugs to target action sites. Nanotechnology increases the efficiency and acceptability of dosage forms by improving their effectiveness, protection, patient adherence and eventually reducing the cost of health care (Ameeduzzafar et al., 2016).

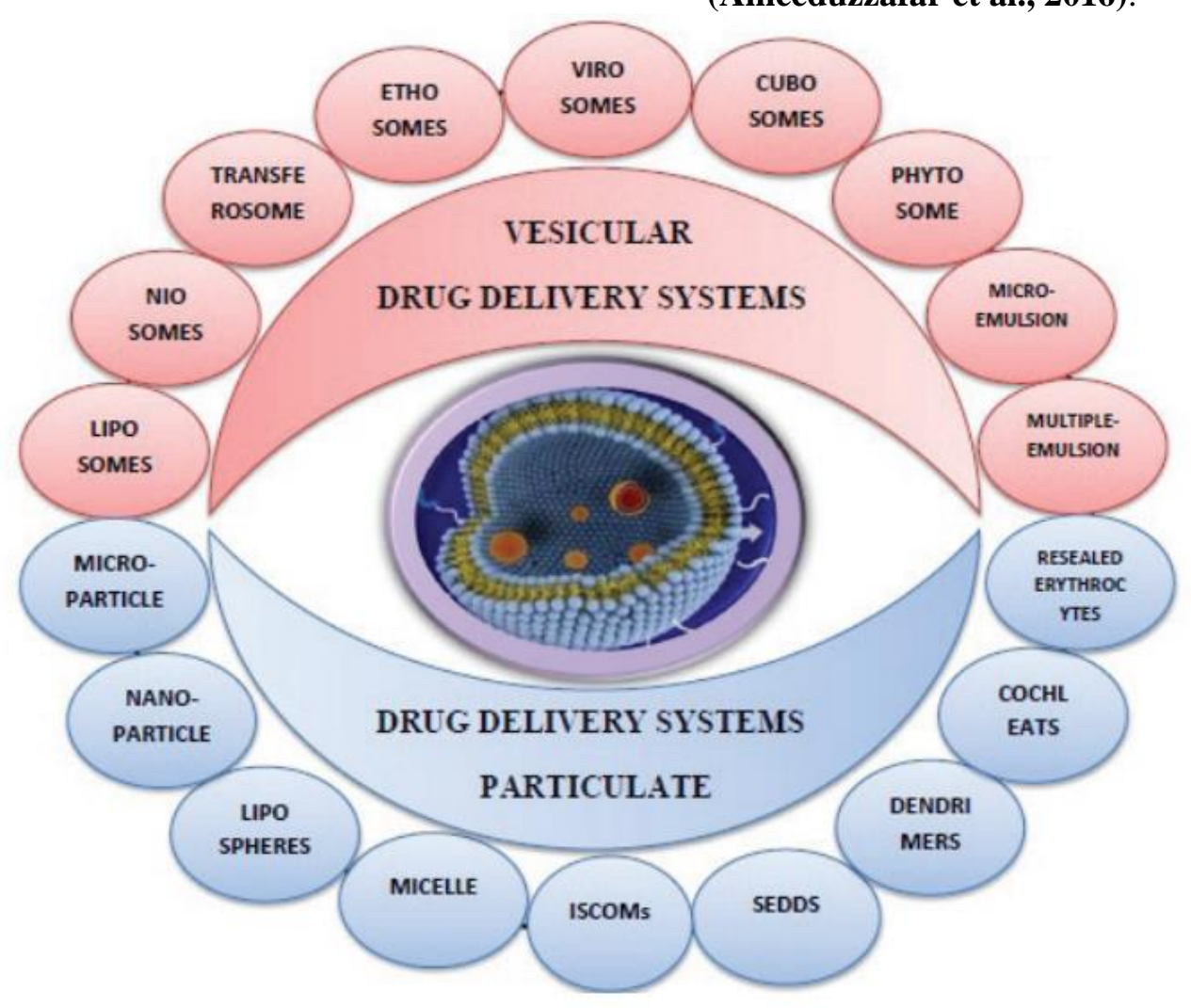

Figure 1: Classification of colloidal drug delivery system (Garg et al., 2015) 
2. Types of colloidal drug delivery system

A-Vesicular drug delivery system.

B-Particulate drug delivery system.

Vesicular structures are tightly ordered assemblies of one or more condensed lipid bilayers produced when exposed to water. Vesicular structures offer many advantages, including drug prolongation in the systemic circulation, reducing toxic hazards, improving the drug targeting, and bioavailability of poorly soluble drugs. Both forms of drugs (hydrophilic or lipophilic) may be introduced, thus preventing the removal of quickly metabolizing drugs and exhibiting persistent release activity (Garg et al., 2015). Such devices tackle medication insolubility, volatility, accelerated deterioration, and high-cost issues. These devices, however, suffer from significant restrictions such as poor drugcharge performance due to critical leakage and reduced stability (Cosco et al., 2008). A variety of vesicular drug delivery systems were subsequently developed: liposomes, niosomes, transferosomes, cubosomes, ethosomes, virosomes, phytosomes, microemulsions, multiple emulsions, etc (Garg \& Goyal, 2014).

\subsection{Liposome}

Liposomes are assemblies formed by cones, composed of one or multiple bilayer lipid membranes. Wilczewska et al. reported that, in the presence of appropriate solvents, phospholipids have bilayered sheaths that eventually twist to shape unilamellar or multilamellar vesicles (Wilczewska et al., 2012). Recently, liposomes have been used as a basic cell model to research the functions and pathways of transport and the properties of penetration. Liposomes exhibit unique properties owing to the amphiphilic existence of the lipids, which render them ideal for the transmission of drugs (Swaminathan \& Ehrhardt, 2012). Phosphatidylcholines (PC) are the lipids that are most commonly used to make liposomes. At all relevant PCs are zwitterionic and may shape lamellar assemblies. The presence of cholesterol has a significant effect on the liposomal characters. Cholesterol acts as a barrier to fluidity, so all the gaps and deficiencies can heal immediately. When films with thin lipids are hydrated, liposomes are formed (Iqbal et al., 2012). Liposomes are categorized into seven major groups depending on structure and application: normal, cationic, long-circulating or stealeth, immuno-related, $\mathrm{pH}$-sensitive, magnetic, and heat-sensitive (Chen et al., 2013).
Liposomes have been prepared using different approaches such as extrusion, micro-emulsification, hand shaking, sonication, dried reconstituted vesicles, freezing-thaw sonication, ethanol injection, ether injection, rapid solvent exchange, de-emulsification, reverse phase evaporation to produce different types. Liposomes have the advantage that their constituents (lecithin and cholesterol) are materials with good biocompatibility which occur naturally (Garg et al., 2015). We can deliver medication to a particular target location within the body and thereby enhance its clinical effectiveness. Liposomes can act as a drug depot which slowly releases the drug over time to maintain therapeutic drug levels (Al-jamal \& Kostarelos, 2011). Despite the benefits of liposomes, they suffer from certain constraints such as reduced stability (phospholipid oxidation), short half-life, high manufacturing costs, leakage and fusion (Vanic et al., 2014).

\subsection{Niosomes}

These are nonionic surfactant-based vesicles produced by nonionic surfactant hydration (alkyl or dialkyl polyglycerol ether class), cholesterol, and certain lipids (Goyal et al., 2013). (Handjani-vila et al. 1979) found them back in the 17th century. Nonionic surfactants form a closed bilayer vesicle structure in aqueous media; hydrophobic fragments are focused away from the aqueous solvent; however, the hydrophilic heads remain in contact with the aqueous solvent after applying heat and agitation. The vesicle, therefore, encapsulates hydrophilic medicines in the enclosed space (ElRidy et al., 2011). Throughout the vesicles, such as van der Waals and repulsive forces, other variables influence the properties of niosomes: structure, particle size, surface charge, concentration, and forces function. The stability of the niosomes is mainly influenced by surfactant form, product existence, storage temperature, charged molecule presence (Garg et al., 2015).

Niosomes may be prepared using different techniques: injection of alcohol, shaking of palms, sonication, extrusion of membranes, evaporation of the reverse step of production from proniosomes (Dufes et al., 2000). In addition to amphiphilic drug moieties with improved therapeutic index, niosomes can encapsulate both hydrophilic and lipophilic drugs. They are stable and act as a depot for the gradual and controlled release of the drug. Various administration routes, including oral, parenteral, or topical routes with increased bioavailability (ElRidy et al., 2011). 


\subsection{Transferosomes}

They were first discovered by Gregor Cevc in 1991. Transfersome is a term recorded as a brand by the German company IDEA as an appropriate drug delivery approach. It is related to the Latin word transferred, which means "to carry across," and the Greek word soma, meaning for a "body"; collected together, they mean "the carrying the body." Besides they are recognized as flexible/ deformable or elastic vesicles.

These are colloidal vesicles, containing a water nucleus of bilayered structured lipids and surfactants (Modi \& Bharadia, 2012). Transferosomes can absorb both hydrophilic, amphiphilic, and lipophilic drug moieties. Such vesicles may create one or more condensed bilayers that are ideal for selective drug distribution regulation. They consist of two principal phosphatidylcholine and surfactant components. After carrier application, transferosomes can squeeze themselves and penetrate by "osmotic gradient." This strong vesicle versatility makes the entry in a selfadapted manner due to the mechanical tension adjacent to it (Patel \& Parikh, 2012).

Transferosomes are processed using four essential techniques such as rotary shaking, hand shaking, sonication, and freezing thaw. These carriers are much more efficient in conveying systemic as well as the topical route to a low and high molecular weight drug. Such vesicles can deform and move through narrow pore sites providing better penetration (Modi \& Bharadia, 2012). Such vesicles protect the encapsulated drug from deterioration and serve as a buffer for slow and regulated emancipation of the substance. They are biocompatible and biodegradable vesicles with good quality of trapping of with small to moderate molecular weight therapeutics (Modi \& Bharadia, 2012). Such carriers provide many benefits such as toxicity deficiency, medication selection, capacity to deliver continuous drug release activity, and simple to scale up. However, owing to the usage of natural polymers, certain device drawbacks are high processing prices, material volatility, risk of degradation, and a deficit of purity (Garg et al., 2015).

\subsection{Ethosomes}

These are non-invasive distribution vesicles or soft vesicles that facilitate the systemic dissemination of deep skin penetration of the medications.
The ethosomal vesicles consist mainly of phospholipids (mostly phosphatidyl choline, phosphatidylserine or phosphatidic acid), alcohol (usually high concentrations of ethanol or isopropyl alcohol), and water (Garg et al., 2014). Touitou first found and researched ethosomes, and named them. The size of the ethosome can be regulated between $30 \mathrm{~nm}$ and a few microns. Ethosomes quickly reach the skin layers leading to higher transdermal efflux. The ideal phospholipid and alcohol concentrations which improve the permeation of ethosomal vesicles have been used effectively. They can clog either hydrophilic, lipophilic, or amphiphilic drugs. The key benefits for ethosomes include strong medication acceptance, increased product permeation, lack of toxicity, and drug distribution usability. Ethosomes are ideal for a range of medicinal applications as well as for biotechnology, agricultural, and beauty applications (Ainbinder et al., 2010). This nano-carrer is non-invasive and eligible for immediate marketing. Ethosomes, however, have drawbacks due to the involvement of ethanol creating instability in the arrangement of skin layers, low yield, and coalescence due to ineffective shell closure (Cortesi et al., 2010).

\subsection{Cubosomes}

These are distinct, nanostructured structures from the crystalline bi-continuous cubic liquid process. Larsson invented the word Cubosomes, which refers to self-assembled liquid crystalline nano-particles of some surfactants with proper water ratio. Cubosomes are nanoparticles that offer uniquely practical properties (Rao et al., 2018).

Some cubosomes comprise primarily of polymers, lipids, and surfactants comprising polar and nonpolar elements, and are thus known to be amphiphilic. By the hydrophobic influence, these amphiphilic molecules are pushed into a polar solvent to spontaneously assemble into nanometersized liquid crystals. Furthermore, these are identical to liquid crystalline material of cubic and viscous crystallography. This cubic process will separate the colloidal and thermodynamically stable dispersions and shape them. In nano drug formulations, cubosomes play a major role by hydrating a mixture of glycerol monooleate and poloxamer 407(Spicer, 2004). They are Squareshaped, $10-500 \mathrm{~nm}$ in diameter somewhat spherical. In cubosomes, drugs are attached via chemical bonds to the polar head of the 
phospholipids. Cubosomes are categorized into two major categories: liquid and powdered cubosomes. Cubosomes are produced using two main techniques: top-down and bottom-up techniques. Top-down technology puts high energy into practice. All strategies include a colloidal stabilizer (Poloxamer 407) to prevent the accumulation of cubosomes (Shen et al., 2013). Usually, cubosomes are created by high energy dispersion of a bulk cubic phase, followed by colloidal stabilization by polymeric surfactants. After the creation of cubosomes the dispersion is shaped into an object. Its unique structure is biologically compatible and able to control the release of drugs. Cubosomes used in skin, hair, etc. treatments; They offer a large surface area and low viscosity and are able of encapsulating hydrophilic and hydrophobic drug molecules (Uyama et al., 2009). Cubosome drug delivery systems have many benefits, including high drug packaging, comparatively easy processing procedure, biodegradable lipids, and managed drug release. While disadvantages involve high-priced technologies, hard to scale, and harm to heatsensitive molecules such as proteins (Rattanapak et al., 2012).

\subsection{Virosomes}

They are round, bilayer phospholipid vesicles with a scale of $120-200 \mathrm{~nm}$ in diameter. The influenza virus is most widely used in the manufacture of virosomes, but even virosomes may be produced from EpsteinBarr virus, HIV, murine leukemia virus, herpes simplex virus, etc. (Moser et al., 2013).

Virosomes contain reconstituted, hollow influenza virus envelopes (70 percent of the virosomal form in phospholipids and phosphatidylcholine); They can not reproduce but are fusion active vesicles containing glycoproteins in the functional envelope (Zurbriggen, 2003). Virosomes bind through ligands to the selected cell, then taken up by endocytosis mediated by the receptor. This results in drug release into the cytosol of the cells. There are several benefits to these vesicles: they are fully biodegradable; they can be added by nasal or parenteral pathway. They are ideal for both babies and immune-depressed individuals (Zurbriggen, 2003). Virosomal delivery systems provide a strong safety design, have patent product protection, and have been licensed in humans. Some drawbacks, however, are an expense, difficulties in preserving long-term stability, and problems in preparing and handling (Khoshnejad et al., 2007). Virosomes used as a delivery platform for vaccines; for example, influenza inflex vaccines and hepatitis A Epaxal and HIV / AIDS mimetics vaccines (Gluck et al., 2005).

\subsection{Phytosome}

The phytosome method was first introduced by Indena S.p. An (Italy) by incorporating phospholipids into the typical plant extract, which increases their absorption and utilization. They are prepared by reacting phospholipids (synthetic or natural) in a ratio of 1:1 on the typical plant extract. Phytosomal particle scale diverges from $50 \mathrm{~nm}$ to several hundred micrometers (Tripathy et al., 2013).

Phosphatidylcholine, phosphatidylethanolamine, and phosphatidylserine, which are derivatives of palmitic, stearic, oleic, or linoleic acid, were the most common phospholipids chosen for phytosome preparation (Di Pierro et al., 2009). Phytosomal physical and chemical properties are determined by particle drsize, permeability, and efficacy of trapping drug. Phytosomes have the following advantages: improved absorption of oral and topical Phyto-components, improved bioavailability, improved therapeutic assistance, reduced dose requirements, improved absorption of the active component, improved stability outline, and proven nutrient safety (Tripathy et al., 2013).

While phytosomes are correlated with certain constraints such as the rapid removal of the constituent from the phytosome and the limited range of action. There are several phytosomal items on the market, such as Ginkoselect capsules, green tea capsules, grapeseed capsules, Panax ginseng capsules, hawthorn soft-gel capsules, and capsules of milk thistle (Lu et al., 2019).

\subsection{Bilosomes}

These are an advanced colloidal distribution mechanism engineered for bile salts to be inserted into liposomes. The involvement of bile salts increases liposomal resilience (Cui et al., 2015). They are more flexible, elastic, and extremely deformable than conventional liposomes. They were invented by Mann et al. in 2004, who established and stabilized vesicles with liposome-like structures (Bashyal et al., 2018).

These novel nanovesicles have several benefits because they improve antigen effectiveness which is poor when injected. The use of live pathogens is 
not required; thus, they are healthy and efficient relative to conventional vaccines. So, they are noninvasive and deliver excellent patient support and compliance. They are suitable for a wide variety of treatments and prolong patent life (Chilkawar et al., 2015).

Sodium glycocholate (SGC), sodium taurocholate (STC), deoxycholic acid (DC), and sodium deoxycholate (SDC) are the most popular bile salts used to prepare bilosomes. Bile salts are endogenous surfactants that are commonly used as diffusion enhancers to increase the transfer of medicines through biological membranes including buccal, neck, intestine, cornea, and blood-brain barriers (ElNabarawi et al., 2019).

The most common bile salts used in the preparation of bilosomes are sodium deoxycholate (SDC), sodium taurocholate (STC), deoxycholic acid (DC), and sodium glycocholate (SGC). Bile salts are endogenous surfactants extensively used as absorption enhancers to improve drug transportation across the biological membranes including buccal, skin, intestinal, cornea, and blood-brain barrier (ElNabarawi et al., 2019). This penetration enhancement occurs through enhancing the solubility of hydrophobic drugs and enhancing the fluidity of both the apical and basolateral membranes (Kesarwani \& Gupta, 2013). Many bilosome based vaccine formulation was prepared as tetanus toxoid vaccine, Bovine serum albumin vaccine, hepatitis $B$ vaccine and influenza vaccine (Chilkawar et al., 2015) Also, the application of bilosomes have been effectively inspected in the transdermal/cutaneous delivery of drugs as diacerein (Aziz et al., 2018), tenoxicam (Al-Mahallawi et al., 2015), buccal delivery of insulin (Bashyal et al., 2018) and oral delivery of cyclosporin A (Guan et al., 2011).

\subsection{Spanlastics}

These are vesicles consisting of span 60 as nonionic surfactants with a nano-sized set of edge activator. The edge activator includes certain hydrophilic surfactant molecules that enable flexibility to the spanlastic lipid bilayer membranes by making pores and producing lipid bilayer destabilization and, in effect, increasing vesicle deformability (Tayel et al., 2015).

They are vesicles composed of span 60 as non-ionic surfactants with an edge activator in a nano-sized range. The edge activator involves other hydrophilic surfactant moieties that afford flexibility to the spanlastic lipid bilayer membranes by making pores and producing destabilization of the lipid bilayers
, and in turn, increase the deformability of the vesicles (Tayel et al., 2015).

Spanlastics, were first developed by Kakkar and Kaur as a novel nano-vesicular carrier for medicines. These vesicles are elastic and have been used in skin distribution of anti-fungal medications to improve the permeability of the cornea. Similar to liposomes and niosomes, spanlastic systems are spherical shaped structures consisting of amphiphilic molecules that function as appropriate media for bioencapsulation (Balakrishnan et al., 2009). They are novel colloidal drug carrier delivery systems intended to deliver drugs in a controlled manner to attain improved patient compliance, specific drug targeting at the site of action, diminish or avoid side effects and more efficient administration of drugs by various routes and for better therapeutic effect (Khositsuntiwong et al., 2012). They are more flexible vesicles that can squeeze themselves to penetrate through the biological membranes. Spanlastics can be used to deliver both hydrophilic and hydrophobic drugs which are encapsulated in the interior hydrophilic compartment and outer lipid layer respectively. It can act as a local depot for sustained release of active compounds (Kaur et al., 2012).

Mechanism of penetration of spanlastics

The current studies propose that the mechanism of penetration of the spanlastic vesicles into the deeper strata is due to a combination of two mechanisms: (Tayel et al., 2015).

Spanlastic vesicles can squeeze themselves through the pores and remain intact after penetration, therefore facilitating the penetration of the drug molecules.

2. Spanlastics act as penetration enhancers, disrupting the highly organized inter-cellular lipids of the biological membrane, and thus penetrating deeply.

Depending on the nature of the active substance (lipophilic or hydrophilic) and the composition of the spanlastics, one of the two mechanisms prevails. Spanlastics are prepared by two methods:

1- Ethanol injection method : (Kakkar \& Kaur, 2011)

The surfactants and drugs are dissolved in ethanol and injected slowly into an aqueous phase, which is heated above the boiling point of the organic solvent. This produces large unilamellar vesicles, which are further treated for size reduction.

2- Thin-film hydration method (TFH) (Shamma et al., 2019) 
It is a simple preparation method and is widely used. In this method, the surfactants are dissolved in an organic solvent in a rounded bottom flask. Then the thin film is formed on the inside wall of the flask by removing the organic solvent using a rotary vacuum evaporator. An aqueous solution such as water or PBS (phosphate buffer saline) [containing drug] is added and the dry film is hydrated above the transition temperature (Tc) of the surfactant resulting in the formation of multilaminar vesicles (MLVs).

\subsection{Arecheosomes}

They are carriers whose construction is made of archaeo bacterial sheath lipids involving di-ether or tetra-ether. They are biocompatible, decomposable and non-toxic in in vivo circumstances (Alavi et al., 2014).

\subsection{Colloidosomes}

Colloidosomes are microcapsules whose shells are composed of colloidal particles. They have established significant attention because of their latent importance in the area of microencapsulation (Thompson et al., 2014). Microencapsulation permits the controlled release of active ingredients in various industrial divisions as medicine, food, and personal care products, allowing the delivery of a range of active moeities such as drugs, pesticides and fragrances. colloidosomes are created by the selfassembly of colloidal particles at the interface between two immiscible liquids, classically water and oil. The original self-assembled assemblies are known as Pickering emulsions (Pickering, 2001). Colloidal particles, such as silica sols and polystyrene latexes have been revealed to be effective Pickering emulsifiers. The preparation of colloidosomes necessitates a mechanism for shell strengthening at the interface to convert the Pickering emulsion precursor into full-bodied microcapsules that can continue removal of the oil/water template (Binks et al., 2005). Colloidosomes were prepared by various techniques as thermal annealing technique, polyelectrolyte complexation and layer-by-layer deposition technique, gel trapping technique, polymerization after Pickering emulsion formation andcovalent cross-linking technique (Thompson et al., 2014).

\subsection{Pharmacosomes}

They are defined as a neutral molecule with positive and negative charges, water-loving and fat-loving properties, and an optimum polyphenol-to- phospholipid ratio in a complex shape. The drugs are present in a dispersion form in this lipoid drug delivery msystem coupled with electron pair sharings and electrostatic forces or by the formation of a hydrogen bond with lipids (Semalty et al., 2009).

Pharmacosome is derived from the term "Pharmakon" which means drug and "soma" meaning carrier. It means a vesicular system where the drug is accompanied with the carrier. These lipid conjugated vesicles may occur as micelles, vesicles, or as colloidal, nanometric sizes in the form of a hexagonal assembly enjoying a functional hydrogen atom banking on the complex architecture. The drug molecule with a free carboxylic or reactive hydrogen atom such as amino, hydroxyl groups, is transformed into an ester with the help of the lipid's hydroxyl moiety, resulting in formation of a prodrug (Pandita \& Sharma, 2013). The prodrug owns both hydrophilic and lipophilic properties. In spite of these properties, prodrugs have the ability to diminish interfacial tension, increase the contact area, and henceforward enhance bioavailability. They assist the transportation through the cell membrane, cell wall, and tissues. If the concentration is increased beyond a level, it may be in a transitional state between liquid and crystal. Such prodrugs assemble into one or more layers after interaction with water, resulting in the creation of pharmacosomes. This method is established by taking into account both the surface properties and the bulk properties of the drug-lipid conjugate

Upon contact with water, these prodrugs assemble into a single or multiple layers resulting in the formation of pharmacosomes. This system is developed by keeping the surface properties as well as the bulk properties of the drug-lipid conjugate in consideration. Pharmacosomes can be prepared either by solvent evaporation technique or ether injection technique (Pandita \& Sharma, 2013).

Relevant Features of Pharmacosomes (Pandita \& Sharma, 2013).

(a) The stability of the entire system is controlled by the physical and chemical characters of the conjugate.

(b) They are easily pass through the cell membrane, walls, or tissues, as they entail both water-loving and fat-loving properties, either by the action of endocytosis or exocytosis.

(c) The rate of degradation depends on size, type of 
the drug functional group, fatty acid chain length in lipids, existence, or absence of spacer. these factors can be diverse to enhance in vivo pharmacokinetic behavior.

(d) They can be offered for administration via a nasal, dental, oral, topical, extra or intravascular pathway.

\subsection{Sphingosomes}

Sphingosome can be defined as "concentric, bilayered vesicle in which an aqueous volume is enclosed entirely by a membranous lipid bilayer consisting mainly of natural or synthetic sphingolipid". Liposomal formulations dependent on sphingo myelin based cholesterol have some benefits compared with other types

Sphingosome may be defined as "concentric, bilayered vesicle in which an aqueous volume is entirely enclosed by a membranous lipid bilayer mainly composed of natural or synthetic sphingolipid". Liposomal formulation based on sphingo myelin based cholesterol has several advantages when compared to other formulation (Singh et al., 2006). The sphingosomes are also more resistant against acid hydrolysis, and have stronger properties of substance retention. Sphingosomes are administered in many ways, including intravenous, intramuscular, subcutaneous and intra-arterial parental and by inhalation route (Sreekanth N, 2013).

General advantages of sphingosomes (Singh et al., 2006).

1. Offer discriminating passive targeting to tumor tissue.

2. Increase therapeutic efficiency.

3. Improve stability through encapsulation.

4. Reduce toxicity of the encapsulated agent.

5. Increase circulation time, thus enhance pharmacokinetic effect .

6. It reduces the therapy cost by imparting improved biopharmaceutical properties to the drug, resulting in improved bioavailability, particularly in case of poorly soluble drugs.

Methods of preparation of sphingosomes:

(Sreekanth N, 2013)

1. Lipid film formation (Hand shaking method).

2. Micro fluidization.

3. Reverse phase evaporation.

\subsection{Layerosomes}

These are typical liposomes that have been coated to protect their shape with one or more layers of biocompatible polyelectrolytes.
The formulation strategy is based on an alternative coating procedure for positive poly(lysine) (pLL) and negative poly(glutamic acid) (pGA) polypeptides on small unilamellar liposomes, which were initially charged. The principal disadvantage of liposomes is their instability in biolgical membrane or during storage due to properities of its surface. This surface alteration stabilized the liposomal structure and led to stable drug delivery systems. Among the potential fields of application are oral administration or incorporation into biomaterials (Bansal et al., 2012).

\subsection{Ufosomes}

They are liposomes of unsaturated fatty acids. Fatty acid vesicles are colloidal solutions of closed lipid bilayers made up of fatty acids and their ionized (soap) molecules. They are observed in a small region within the ternary phase fatty acid-soapwater diagram above the melting chain temperature (Tm) of the corresponding fatty acid-soap mixture54. Fatty acid vesicles often produce two forms of amphiphiles, the non-ionized neutral form and the ionized form (the soap filled with negative effect). For vesicle stability the ratio of nonionized neutral phase and ionized phase is important. Fatty acid vesicles are actually "fatty acid / soap vesicles" mixed together (Bansal et al., 2012).

\subsection{Vesosomes}

They are liposomes encapsulating small liposomes (multicompartment system). Vesosomes consist of liposomes of various sizes that are biocompatible and biodegradable. This novel drug delivery system offers double protection to encapsulated drugs, one of which is the two-layer of encapsulated small liposomes, while the other is the bilayer of the larger liposomes enclosing the smaller. Another important advantage in multicompartment systems is that a medication mixture, which in certain situations is made in non-compatible products, may be encapsulated in the internal liposomal. And, in contrast with single-compartment systems, medication release from such multicompartment systems may be controlled more efficiently (Paleos et al., 2013).

\subsection{Invasomes}

They are novel vesicles incorporating terpenes with enhanced penetration compared to the conventional liposomes. These are soft liposomal vesicles with very high membrane fluidity, containing terpenes, which are playing the role of penetration enhancement. The presence of terpenes and ethanol 
makes invasomes unique. These vesicles have shown to possess the combined advantages of liposomes, which are potential carriers and penetration enhancement of the terpenes, which are having the ability to modify the order of SC packing thus promoting skin delivery (Lakshmi PK, Kalpana B, 2013).

\section{Advantages}

- Non-invasive system of medication delivery.

- Improved drug permeation via the skin for transdermal drug delivery.

- It is possible to deliver hydrophilic or lipophilic drugs.

- Encompasses non-toxic raw material in their formulation.

- High patient compliance (drug administered as semisolid form gel or cream).

- Simple technique for drug delivery compared to iontophoresis and phonophoresis.

\section{Solid lipid nanoparticle (SLN)}

Since 1990, SLNs have been presented as a substitute carrier system for liposomes, emulsions, and polymeric nanoparticles.17 They have an average size of 40 to $1000 \mathrm{~nm}$ and a spherical shape which can be deliberated with transmission electron microscopy and scanning electron microscopy (Thatipamula et al., 2011)

SLNs consist of around $0.1-30$ (percent $\mathrm{w} / \mathrm{w}$ ) solid fat that is spread in an aqueous environment. To improve stability, surfactants are used in concentrations of around 0.5 to 5 percent. The correct collection of lipids and surfactants will influence particle size, long-term storage stability, medication loading and release behaviour.19 Their lipid components are stable at both body and ambient temperature (Blasi et al., 2007).

SLNs have many advantages compared to other methods, including ease of preparation, low expense, strong efficiency, excellent physical durability, reasonable release profile, chemical flexibility, organic solvent-free preparation, no lipid carrier toxicity, lipid biodegradability, cheaper than polymer carrier, better clearance and reliability, and biodegradability (Lakshmi PK, Kalpana B, 2013). 19. Nano structure lipid carrier (NLC)

NLCs are modified SLNs in which lipidic phase is consist of both solid (fat) and liquid (oil) lipids at ambient temperature that improves the stability and drug loading capacity. NLCs present in three forms structure. The first type is imperfect type in which solid and liquid fats (oil) are mixed in various lipid structures. Imperfect lipid matrix arranged with a distance between triglyceride fatty acid chains in crystal, thus increasing the capacity of the drugs to join the matrix. The second class is formless category (non-crystalline matrix), which is a category of NLC that has no crystalline structure and therefore avoids the expulsion of an amorphous loaded material. The third type is multiple form in which the solubility in liquid lipid is greater than in solid lipid, while preserved by solid lipid from decomposition. This type of NLCs is identical to the emulsions w / o / w.(Lakshmi PK, Kalpana B, 2013).

\section{Conclusion}

Colloidal drug delivery systems play a significant role in drug delivery. In my opinion, all colloidal carriers present notable characteristics after application. These vesicles can deliver an active compound through the biological membranes via absorption, fusion, and to raise the quantity of permeated drugs compared with conventional formulations.

\section{Declaration of interest}

The authors declare that they have no conflicts of interest concerning the preparation of this manuscript.

\section{References}

Ainbinder, D., Paolino, D., Fresta, M., \& Touitou, E. (2010). Drug delivery applications with ethosomes. Journal of Biomedical Nanotechnology, 6(5), 558-568.

Al-jamal, W. T., \& Kostarelos, K. (2011). Liposomes: From a Clinically Established Drug Delivery System to a Nanoparticle Platform for Theranostic Nanomedicine. 44(10).

Al-Mahallawi, A. M., Abdelbary, A. A., \& Aburahma, M. H. (2015). Investigating the potential of employing bilosomes as a novel vesicular carrier for transdermal delivery of tenoxicam. International Journal of Pharmaceutics, 485(1-2), 329-340.

Alavi, S. E., Mansouri, H., Esfahani, M. K. M., Movahedi, F., Akbarzadeh, A., \& Chiani, M. (2014). Archaeosome: As new drug carrier for delivery of paclitaxel to breast cancer. Indian Journal of Clinical Biochemistry, 29(2), 150-153.

Ameeduzzafar, A., Ali, J., Fazil, M., Qumbar, M., Khan, N., \& Ali, A. (2016). Colloidal drug delivery system: Amplify the ocular delivery. Drug Delivery, 23(3), 710-726. 
Aziz, D. E., Abdelbary, A. A., \& Elassasy, A. I. (2018). Investigating superiority of novel bilosomes over niosomes in the transdermal delivery of diacerein: in vitro characterization, ex vivo permeation and in vivo skin deposition study. Journal of Liposome Research, 29(1), 73-85.

Balakrishnan, P., Shanmugam, S., Lee, W. S., Lee, W. M., Kim, J. O., Oh, D. H., Kim, D. D., Kim, J. S., Yoo, B. K., Choi, H. G., Woo, J. S., \& Yong, C. S. (2009). Formulation and in vitro assessment of minoxidil niosomes for enhanced skin delivery. International Journal of Pharmaceutics, 377(1-2), 18.

Bansal, S., Prasad Kashyap, C., Aggarwal, G., \& Harikumar, S. (2012). Ijrpc 2012, 2(3) International Journal of Research in Pharmacy and Chemistry a Comparative Review on Vesicular Drug Delivery System and Stability Issues. Ijrpc, 2(3), 704-713. www.ijrpc.com

Bashyal S, Seo J, Keum T, Noh G, C. Y. (2018). Facilitated permeation of insulin across TR146 cells by cholic acid derivatives-modified elastic bilosomes. International Journal of Nanomedicine, 13, 5173-5186.

Binks, B. P., Murakami, R., Armes, S. P., \& Fujii, S. (2005). Temperature-induced inversion of nanoparticle-stabilized emulsions. Angewandte Chemie - International Edition, 44(30), 4795-4798.

Blasi, P., Giovagnoli, S., Schoubben, A., Ricci, M., \& Rossi, C. (2007). Solid lipid nanoparticles for targeted brain drug delivery. Advanced Drug Delivery Reviews, 59(6), 454-477.

Chen, M. H., Soda, Y., Izawa, K., Kobayashi, S., Tani, K., Maruyama, K., Tojo, A., \& Asano, S. (2013). A versatile drug delivery system using streptavidin-tagged pegylated liposomes and biotinylated biomaterials. International Journal of Pharmaceutics, 454(1), 478-485.

Chilkawar, R. N., Labs, M., Nanjwade, B. K., Life, T., Pvt, S., \& Idris, S. (2015). Bilosomes Based Drug Delivery System. Journal of Chemistry and Applications, 2(1).

Cortesi, R., Romagnoli, R., Drechsler, M., Menegatti, E., Zaid, A. N., Ravani, L., \& Esposito, E. (2010). Liposomes- and ethosomes-associated distamycins: A comparative study. Journal of Liposome Research, 20(4), 277-285.

Cosco, D., Celia, C., Cilurzo, F., Trapasso, E., \& Paolino, D. (2008). Colloidal carriers for the enhanced delivery through the skin. Expert Opinion on Drug Delivery, 5(7), 737-755. https://doi.org/10.1517/17425247.5.7.737

Cui, M., Wu, W., Hovgaard, L., Lu, Y., Chen, D., \& Qi, J. (2015). Liposomes containing cholesterol analogues of botanical origin as drug delivery systems to enhance the oral absorption of insulin. International Journal of Pharmaceutics, 489(1-2), 277-284.

de la Fuente, M., Raviña, M., Paolicelli, P., Sanchez, A., Seijo, B., \& Alonso, M. J. (2010). Chitosanbased nanostructures: A delivery platform for ocular therapeutics. Advanced Drug Delivery Reviews, 62(1), 100-117. https://doi.org/10.1016/j.addr.2009.11.026

Di Pierro, F., Menghi, A. B., Barreca, A., Lucarelli, M., \& Calandrelli, A. (2009). GreenSelect ${ }^{\circledR}$ Phytosome as an adjunct to a low-calorie diet for treatment of obesity: A clinical trial. Alternative Medicine Review, 14(2), 154-160.

Dufes, C., Schätzlein, A. G., Tetley, L., Gray, A. I., Watson, D. G., Olivier, J. C., Couet, W., \& Uchegbu, I. F. (2000). Niosomes and polymeric chitosan based vesicles bearing transferrin and glucose ligands for drug targeting. Pharmaceutical Research, 17(10), 1250-1258.

El-Nabarawi, M. A., Shamma, R. N., Farouk, F., \& Nasralla, S. M. (2019). Bilosomes as a novel carrier for the cutaneous delivery for dapsone as a potential treatment of acne: preparation, characterization and in vivo skin deposition assay. Journal of Liposome Research, 30(1), 1-11.

El-Ridy, M. S., Abdelbary, A., Essam, T., Abd ElSalam, R. M., \& Aly Kassem, A. A. (2011). Niosomes as a potential drug delivery system for increasing the efficacy and safety of nystatin. Drug Development and Industrial Pharmacy, 37(12), 1491-1508.

Garg, T., \& Goyal, A. K. (2014). Biomaterial-based scaffolds-current status and future directions. Expert Opinion on Drug Delivery, 11(5), 767-789.

Garg, T., Rath, G., \& Goyal, A. K. (2014). Comprehensive review on additives of topical dosage forms for drug delivery. Drug Delivery, 22(8), $1-19$.

https://doi.org/10.3109/10717544.2013.879355

Garg, T., Rath, G., \& Goyal, A. K. (2015). Colloidal

Drug Delivery Systems : Current Status and Future Directions. 32(2), 89-147.

Goyal, G., Garg, T., Malik, B., Chauhan, G., Rath, G., \& Goyal, A. K. (2013). Development and 
characterization of niosomal gel for topical delivery of benzoyl peroxide. 7544(2010), 1-16.

Guan, P., Lu, Y., Qi, J., Niu, M., Lian, R., Hu, F., \& $\mathrm{Wu}, \mathrm{W}$. (2011). Enhanced oral bioavailability of cyclosporine A by liposomes containing a bile salt. International Journal of Nanomedicine, 6, 965-974. Gupta, H., Aqil, M., Khar, R. K., Ali, A., Bhatnagar, A., \& Mittal, G. (2013). Nanoparticles laden in situ gel for sustained ocular drug delivery. Journal of Pharmacy and Bioallied Sciences, 5(2), 162-165.

Iqbal, M. A., Md, S., Sahni, J. K., Baboota, S., Dang, S., \& Ali, J. (2012). Nanostructured lipid carriers system: Recent advances in drug delivery. Journal of Drug Targeting, 20(10), 813-830.

Kakkar, S., \& Kaur, I. P. (2011). Spanlastics-A novel nanovesicular carrier system for ocular delivery. International Journal of Pharmaceutics, 413(1-2), 202-210.

Kaur, I. P., Rana, C., Singh, M., Bhushan, S., Singh, H., \& Kakkar, S. (2012). Development and Evaluation of Novel Surfactant-Based Elastic Vesicular System for Ocular Delivery of Fluconazole. Journal of Ocular Pharmacology and Therapeutics, 28(5), 484-496.

Kayser, O., Lemke, A., \& Hernandez-Trejo, N. (2005). The Impact of Nanobiotechnology on the Development of New Drug Delivery Systems. Current Pharmaceutical Biotechnology, 6(1), 3-5.

Kesarwani, K., \& Gupta, R. (2013). Bioavailability enhancers of herbal origin: An overview. Asian Pacific Journal of Tropical Biomedicine, 3(4), 253266.

Khoshnejad, M., Young, P., Toth, I., \& Minchin, R. (2007). Modified Influenza Virosomes: Recent Advances and Potential in Gene Delivery. Current Medicinal Chemistry, 14(29), 3152-3156.

Khositsuntiwong, N., Manosroi, A., Götz, F., Werner, R. G., Manosroi, W., \& Manosroi, J. (2012). Enhancement of gene expression and melanin production of human tyrosinase gene loaded in elastic cationic niosomes. Journal of Pharmacy and Pharmacology, 64(10), 1376-1385.

Lakshmi PK, Kalpana B, P. D. (2013). Invasomesnovel vesicular carriers for enhanced skin permeation. Systematic Reviews in Pharmacy, 4(1), 26-30.

Lu, M., Qiu, Q., Luo, X., Liu, X., Sun, J., Wang, C., Lin, X., Deng, Y., \& Song, Y. (2019). Phytophospholipid complexes ( phytosomes ): A novel strategy to improve the bioavailability of. Asian Journal of Pharmaceutical Sciences, 14(3), 265-274. Modi, C. ., \& Bharadia, P. (2012). Transfersomes:
New Dominants for Transdermal Drug Delivery. American Journal of PharmTech Research AJPTR., 2(January 2012), 71-91.

Moser, C., Müller, M., Kaeser, M. D., Weydemann, U., \& Amacker, M. (2013). Influenza virosomes as vaccine adjuvant and carrier system. Expert Review of Vaccines, 12(7), 779-791.

Paleos, C. M., Tsiourvas, D., Sideratou, Z., \& Pantos, A. (2013). Formation of artificial multicompartment vesosome and dendrosome as prospected drug and gene delivery carriers. Journal of Controlled Release, 170(1), 141-152.

Pandita, A., \& Sharma, P. (2013). Pharmacosomes: An Emerging Novel Vesicular Drug Delivery System for Poorly Soluble Synthetic and Herbal Drugs. ISRN Pharmaceutics, 2013(3), 1-10.

Patel, B., \& Parikh, R. H. (2012). Preparation and formulation of transferosomes containing an antifungal agent for transdermal delivery: Application of Plackett-Burman design to identify significant factors influencing vesicle size. Journal of Pharmacy and Bioallied Sciences, 4(SUPPL.), 60-61.

Pickering, 2001. (2001). 72, 156),. 2001-2021.

Rao SW, BN, S., \& K, P. (2018). A review on cubosome: The novel drug delivery system. GSC Biological and Pharmaceutical Sciences, 5(1), 076081.

Rattanapak, T., Young, K., Rades, T., \& Hook, S. (2012). Comparative study of liposomes, transfersomes, ethosomes and cubosomes for transcutaneous immunisation: Characterisation and in vitro skin penetration. Journal of Pharmacy and Pharmacology, 64(11), 1560-1569.

Semalty, A., Semalty, M., Rawat, B. S., Singh, D., \& Rawat, M. S. M. (2009). Pharmacosomes: The lipid-based new drug delivery system. Expert Opinion on Drug Delivery, 6(6), 599-612.

Shamma, R. N., Sayed, S., Sabry, N. A., \& ElSamanoudy, S. I. (2019). Enhanced skin targeting of retinoic acid spanlastics: in vitro characterization and clinical evaluation in acne patients. Journal of Liposome Research, 2104.

Shen, H. H., Lake, V., Le Brun, A. P., James, M., Duff, A. P., Peng, Y., McLean, K. M., \& Hartley, P. G. (2013). Targeted detection of phosphatidylserine in biomimetic membranes and invitro cell systems using annexin V-containing cubosomes. Biomaterials, 34(33), 8361-8369.

Singh, J., Chhabra, G., \& Pathak, K. (2014). Development of acetazolamide-loaded, $\mathrm{pH}$ triggered polymeric nanoparticulate in situ gel for sustained ocular delivery: In vitro. ex vivo evaluation and pharmacodynamic study. Drug 
Development and Industrial Pharmacy, 40(9), 12231232.

Singh, S., Malik, B. K., \& Sharma, D. K. (2006). Molecular drug targets and structure based drug design: A holistic approach. Bioinformation, 1(8), 314-320.

Spicer, P. (2004). Cubosome processing: Industrial nanoparticle technology development. Chemical Engineering Research and Design, 83(11 A), 12831286.

Sreekanth N. (2013). SPHINGOSOMES: A Novel vesicular drug delivery system. 2(2), 305-312.

Swaminathan, J., \& Ehrhardt, C. (2012). Liposomal delivery of proteins and peptides. Expert Opinion on Drug Delivery, 9(12), 1489-1503.

Tayel, S. A., El-Nabarawi, M. A., Tadros, M. I., \& Abd-Elsalam, W. H. (2015). Duodenum-triggered delivery of pravastatin sodium via enteric surfacecoated nanovesicular spanlastic dispersions: Development, characterization and pharmacokinetic assessments. International Journal of Pharmaceutics, 483(1-2), 77-88.

Thatipamula, R. P., Palem, C. R., Gannu, R., Mudragada, S., \& Yamsani, M. R. (2011). Formulation and in vitro characterization of domperidone loaded solid lipid nanoparticles and nanostructured lipid carriers. DARU, Journal of Pharmaceutical Sciences, 19(1), 23-32.
Thompson, K. L., Williams, M., \& Armes, S. P. (2014). Colloidosomes: Synthesis, properties and applications. Journal of Colloid and Interface Science, 447, 217-228.

Tripathy, S., Patel, D. K., Barob, L., \& Naira, S. K. (2013). a Review on Phytosomes, Their Characterization, Advancement \& Potential for Transdermal Application. Journal of Drug Delivery and Therapeutics, 3(3).

Uyama, M., Nakano, M., Yamashita, J., \& Hana, T. (2009). Useful modified cellulose polymers as new emulsifiers of cubosomes. Langmuir, 25(8), 43364338.

Vanic Z Hurler J, Ferderber K, Gašparovic P, \& J, B. N. F.-G. (2014). Novel vaginal drug delivery system: deformable propylene glycol liposomes-inhydrogel. 24(1), 27-36.

Wilczewska, A. Z., Niemirowicz, K., Markiewicz, K. H., \& Car, H. (2012). Nanoparticles as drug delivery systems. Pharmacological Reports, 64(5), 1020-1037.

Zurbriggen, R. (2003). Immunostimulating reconstituted influenza virosomes. Vaccine, 21(910), 921-924. 